
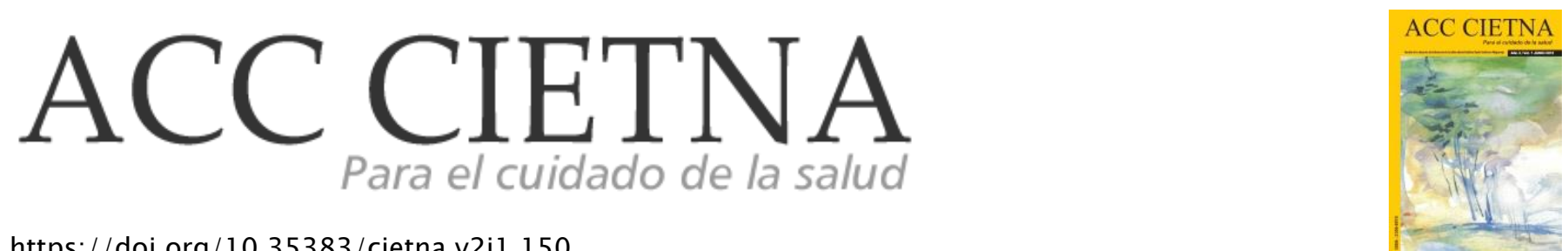

https://doi.org/10.35383/cietna.v2i1.150

\title{
Experiencias de los estudiantes del tercer ciclo de enfermería en el uso del diario de práctica
}

\author{
León Tenorio Libia Magaly ${ }^{1}$, Cervera Vallejos Mirtha Flor ${ }^{2}$
}

\begin{tabular}{l} 
INFORMACIÓN DEL ARTÍCULO \\
\hline Historia del artículo: \\
Recibido el 3 de enero de 2015 \\
Aceptado el 15 de mayo de 2016
\end{tabular}

\section{Palabras claves:}

Experiencias

Diario de práctica

Estudiantes de enfermería

\section{RESUMEN}

La presente investigación cualitativa con abordaje de estudio de caso "Experiencias de los Estudiantes del tercer ciclo de Enfermería en el uso de diario de práctica" tuvo como objetivos: describir y analizar sus experiencias, en el uso de dicho diario. El escenario donde se realizó el estudio fueron las aulas de la escuela de enfermería. La muestra fue obtenida por saturación y lo constituyeron 13 estudiantes del tercer ciclo grupo A, de la asignatura cuidados de enfermería materno perinatal. Los instrumentos de recolección de datos fueron la entrevista Semi estructurada a profundidad y el análisis documental; para el procesamiento de los datos se utilizó el análisis temático. En el proceso de investigación se tuvo en cuenta los principios éticos de la investigación de la ética personalista. Emergieron tres categorías: Experiencias de una herramienta consolidada de práctica con las subcategorías: Para observaciones y anotaciones de la práctica, para el seguimiento en la consolidación de competencias, para estandarizar el lenguaje de enfermería, para autoevaluación del aprendizaje. Registro de sentimientos personales, con las subcategorías: Satisfacción profesional frente a logros escritos. Consolidación de la teoría con la práctica y viceversa.

\footnotetext{
$\overline{1}$ Licenciada en Enfermería. Enfermera con trabajo independiente, Chiclayo, Perú. Email: $\underline{\text { lleon@gmail.com }}$

2 Doctora en Enfermería. Universidad Nacional de Trujillo. Miembro del Instituto de Bioética. USAT. Vicerrectora de Profesores e Investigación. Docente del Master de Bioética y de Enfermería. Asesora de tesis de Maestría de Bioética y Enfermería, Chiclayo, Perú.
} 
Students experiences of third cycle of Nursing in daily practice.

\section{ABSTRACT}

Keywords:

Experiences

Daily of practice

Nursing students.
This qualitative research study addressing case "Experiences of Students of the third cycle of Nursing in the use of daily practice" aimed to: describe and analyze their experiences in the use of the journal. The scene where the study was conducted were the classrooms of the school of nursing. The sample was obtained by saturation and constituted 13 postgraduate students of group $A$, the subject of perinatal maternal care nursing. The data collection tools were semi-structured in-depth interviews and document analysis, processing of the data analysis was used temático. In the research process took into account the ethical principles of research ethics Emerging personalista, three categories: Experiences of an established practice tool subcategories: For comments and annotations practice to monitor the consolidation of powers to standardize nursing language for self- learning. Registration personal feelings, with subcategories: Job satisfaction versus written achievements. Consolidation theory with practice and vice versa.

\section{Introducción}

El estudiante de enfermería va adquiriendo su formación profesional a través del tiempo, esta, exige cinco años de estudios, dados en semestres académicos denominados ciclos lectivos de enseñanza-aprendizaje, donde está inmersa las prácticas hospitalarias y comunitarias, con esto se pretende que el estudiante vaya adquiriendo competencias que lo cualifiquen en el futuro como una enfermera (o) eficiente en la solución de los problemas de salud de las personas.

Las investigadoras en su propia experiencia y en las que narran diferentes autores; percibieron que el proceso de formación del pregrado de enfermería es lento y progresivo en el tiempo donde el estudiante toma conciencia de su ser y hacer profesional, con el desarrollo de procesos cognitivos, habilidades y actitudes, conducentes a adquirir competencias desde las aulas hacia los centros de práctica!.

El desarrollo de estos procesos se evidencia en el desarrollo de competencias que son exigidas en todas las asignaturas troncales de la profesión, es así como en la asignatura cuidados de enfermería materno-perinatal grupo A, los estudiantes percibieron ciertas limitaciones pues algunos de ellos todavía no habían valorado a una gestante o no habían realizados el número de maniobras de Leopold, que deberían hacer y se daban cuenta porque no lo tenían registrado en su diario de práctica.

Este diario, exigido por las profesoras de la asignatura durante la rotación de práctica, era revisado y firmado para ver el cumplimiento de las competencias prácticas, igualmente algunos estudiantes referían no haber cumplido con el número de sesiones educativas a la familia, porque solo tenían registrado en su diario el informe de dos o una visita domiciliaria; también llamó la atención observar como el estudiante a través de su diario fijaba por escrito las palabras propias de la asignatura, definiciones, gráficos nombre de medicamentos, etc. 
En este sentido, las investigadoras al conocer esta actividad enriquecedora realizada por los estudiantes quiso abstraer la experiencia de su uso durante la formación del estudiante de enfermería tanto en el campo comunitario, como hospitalario, ya que otros estudiantes de diferentes ciclos carecían de un diario de práctica, y muchos procedimientos que se enseñaban en aula en forma teórica o con prácticas simuladas, se quedaban sin terminar por no llevar un registro sistematizado a través de un diario. Ante esta realidad percibida se formuló el siguiente problema de investigación, como ¿Cuáles son las experiencias de los estudiantes del tercer ciclo de enfermería de la Universidad Católica Santo Toribio de Mogrovejo (USAT) en el uso del diario de práctica?, teniendo como objeto de estudio, las experiencias de los estudiantes en el uso de este diario. Los objetivos propuestos fueron: Describir y analizar las experiencias de los estudiantes de enfermería del tercer ciclo en el uso de diario de práctica.

La investigación resultó relevante porque no existían investigaciones a nivel nacional y local de esta índole, y se justifica porque el estudiante puede usar un instrumento que potenciará su capacidad de reflexión sobre logros de competencias determinados en el silabo de una asignatura de naturaleza obligatoria.

Por otro lado, los logros escritos en el diario de práctica ${ }^{2,3}$, permitirán realizar una evaluación individual del estudiante recibiendo retroalimentación continua del proceso enseñanza-aprendizaje $\mathrm{y}$ en este proceso la intervención del profesor es de suma importancia, para evaluar los resultados obtenidos después de cada rotación de sus asignaturas troncales donde en conjunto estudiantes y profesores puedan realizar los ajustes y cambios necesarios hacia el logro de las competencias.

Es así como, la investigación permitió hacer una reflexión sobre todo el proceso formativo en sus dos roles, en su centro de estudios y en sus prácticas que a diferencia de otros compromete sus sentimientos, dedicación, afectos que influyeron en la toma de sus decisiones ante los problemas que se presentaron y se dieron cuenta que lograron obtener competencias en su solución a través de un diario de práctica, punto de partida para la profundización de otras investigaciones que busquen y se planteen el mejoramiento de la formación integral de los estudiantes de enfermería.

En la investigación partimos socializando algunos términos y/o definiciones que son importantes mencionar, así tenemos la experiencia que proviene del vocablo experi que significa comprobar o experimentar algo para la adquisición de destrezas, en este sentido, la experiencia es el conocimiento derivado de la observación, de la vivencia que pueda suceder en la vida 4 . Es decir todo aquello que se ha vivido y que le ha ocurrido a alguien ${ }^{5}$

Etimológicamente proviene del vocablo latino esperior (experimentar), y perior (probar., prueba, comprobación y paso a través de un peligro. Así el hombre experimentado (perito) es el que se ha sometido a un paso dificultoso y lo ha superado, adquiriendo destreza para salir airoso en casos parecidos 6 .

Aristóteles, citado por García6, determina que la experiencia es un conocimiento de la sensación, pero inferior al arte y a la ciencia. En cambio Santo Tomas, citado por García6, determina que las experiencias son un conocimiento propiamente de los sentidos, pero se extiende también al entendimiento tanto por lo que hace el conocimiento de las cosas materiales, como por lo que le toca al conocimiento de los propios actos espirituales y de la misma existencia del yo.

Por su parte Reeve ${ }^{7}$ afirma que la experiencia está determinada por el conocimiento derivado de la observación, de la vivencia de un evento que sucede en la vida, se refiere al conocimiento procedimental (como hacer algo), este concepto lo 
reafirma Peplau, en Tomey8, quien expresa que la experiencia es todo aquello que se ha vivido y lo que le ha ocurrido a alguien. A su vez Papalia, ${ }^{9}$ dice que existen experiencias buenas y malas, las cuales son filtradas a través de la personalidad peculiar y la perspectiva de vida, son las personas quienes determinan cuales de estas experiencias serán agradables y cuáles serán desagradables y las hacen en parte por la manera cómo actúan para construir su propia vida, en parte por el modo de reaccionar frente a la forma que se va adquiriendo esta. Manuel Kant citado por García ${ }^{6}$, determina que la experiencia es una síntesis de lo dado a nuestra sensibilidad, toda experiencia contiene, además de la intuición de los sentidos, un concepto de objeto, que está dado o aparece en la intuición, descansa en la unidad sintética de los fenómenos, es decir, es una síntesis según conceptos del objeto de los fenómenos en general, $\sin$ la cual la experiencia no sería ningún conocimiento.

$Y$ para las investigadoras la experiencia con relación a las estudiantes del tercer ciclo en el uso de su diario viene dado por lo vivido durante un semestre al registrar los aspectos relacionados con sus prácticas en una libreta en forma escrita y conforme pasaba el tiempo lo han ido construyendo, adquiriendo de esta manera destrezas, fortaleciendo conocimiento o mejorando sus actitudes, pues los procedimientos vivencia de una práctica sucedida no solo en el escritos eran derivados de la observación, de la hospital sino también en la comunidad ${ }^{10}$.

Quien vive la experiencia es una persona, y en nuestro estudio son las estudiantes de enfermería. Una estudiante es una persona joven que postula a la carrera de enfermería, para lo cual tiene que reunir un perfil determinado, de acuerdo a las exigencias de la escuela de enfermería y de la universidad a la que pertenece. Todo estudiante en sus prácticas, se desarrolla y aplica de forma integral, en el marco, de los cuidados al enfermo, familia y la comunidad en el continuo saludenfermedad 4 . Por ello, enfermería se considera una disciplina erudita de servicio cuyo propósito es contribuir de manera individual y colectiva a promover un nivel funcional óptimo de las personas que reciben cuidado para alcanzar el equilibrio deseado?.

En este sentido, la estudiante de enfermería tiene que dedicarse cada vez más a una variedad de actividades públicas $u$ hospitalarias las cuales influyen en la salud de las personas y en sus capacidades funcionales óptimas.

Independientemente del tipo de personas $y$ centros del que se trate, la enfermera en formación debe conocer y aplicar las distintas técnicas epidemiológicas y de investigación social para conseguir el desarrollo de la comunidad, siguiendo las normas básicas y lineamientos establecidos por el estado o por el establecimiento al que pertenece, conociendo la realidad de la comunidad y del centro hospitalario, para así lograr cumplir los objetivos establecidos en sus horas de prácticas 5 .

Para ello, los estudiantes de enfermería se acercan y mantienen contacto permanente con los grupos sociales, con los hogares, los centros de trabajo, las escuelas, ya que es allí donde se desarrollan íntegramente, y donde se pone de manifiesto las necesidades reales o potenciales de salud, además donde se encuentran la mayoría de los factores de riesgo que inciden negativamente en la salud de las personas. Además, en este acercamiento se va descubriendo las fortalezas que están inmersas en las personas y emergiendo a la luz sus problemas, oportunidad aprovechada por los estudiantes para darles solución con la propia participación de las familias, haciendo uso de sus propios recursos y potencialidades ya sea en recurso humano, materiales o los aportes de su experiencia vivida. De esta manera el estudiante de enfermería contribuye de alguna manera en direccionar, hábitos y conductas que fomenten el autocuidado, los cuales generen el propio desarrollo sostenible, en el marco de la promoción y prevención de la salud. 
Por su parte el diario de práctica, consiste en una libreta de apuntes de 50 a 100 hojas, donde tienen registrado sus datos personales, y conforme van sucediendo sus prácticas en el tiempo, van detallando los procedimientos que ya realizaron.

En otros lugares como la Universidad de Córdova -España ${ }^{3}$ en su escuela de enfermería en las asignatura de Médico Quirúrgico I, en enfermería materno Infantil I y Farmacología Nutrición y Dietética, el diario de práctica es conocido como diario reflexivo porque la estudiante de enfermería recopila, organiza, resume e interpreta la información sobre lo que es objeto de análisis de su práctica, con el propósito de facilitar la toma de decisiones o conducir el proceso educativo a través de acciones adecuadas, tanto para el educador como para la estudiante. En este sentido se pretende que los estudiantes logren los objetivos propuestos en la práctica comunitaria y hospitalaria antes recibida en la teoría desde el aula, de tal manera que se refleje el conocimiento enfermero en ambos mediante el desarrollo de una metodología centrada en el alumno y basada en una práctica reflexiva.

En el diario de prácticas se puede anotar, aspectos relevantes de la práctica y experiencias significativas. Esto exigirá un proceso reflexivo a la vez que la revisión y estudio de dichas notas, que ayudarán al estudiante a realizar consultas bibliográficas y la revisión del material de las clases teóricas. También su contenido le puede ser muy útil para su posterior discusión con la profesora sobre: Experiencias relevantes de la práctica, técnicas realizadas, actividades habituales que hayan dado lugar a una reflexión en la acción, problemas y dificultades, logros obtenidos y sentimientos que se hayan generado durante una práctica concreta o a lo largo de un período ${ }^{2,3}$.

Es esencial que toda la información de las experiencias en las prácticas recopiladas en el diario, preservando la confidencialidad de todas las personas, y si fuera el caso de tomar sus nombres con el fin de la identificación de familias en riesgo, estas serán únicamente tomadas como parte del trabajo y no para otros fines, entonces el diario se convierte en un importante instrumento de investigación, cuya información no debe transferirse a otra persona que no esté autorizada conservándose sus datos en un lugar seguro. Por medio de su ejercicio se intenta que adquieran mecanismos de reflexión sobre las actividades que ejecutan, respaldadas en el conocimiento enfermero, permitiéndoles evaluar las competencias propias de una determinada asignatura, dirigiendo las acciones hacia las causas básicas de la pérdida de salud (higiene, alimentación, riesgos domésticos, etc.), de una forma multisectorial, con la participación de la persona o comunidad misma, poniendo los medios a su alcance para mejorar su salud así como para evitar la enfermedad 5 .

El compromiso individual se concreta con el crecimiento personal, profesional, formación universitaria, especialización, y formación continuada. relacionado con: adhesión a los valores, desarrollo de conocimientos, apoyo a la investigación, solidaridad profesional, participación colectiva, orgullo, socialización para tomar también bien las decisiones concernientes quienes reciben el cuidado.

\section{Metodología}

La investigación fue cualitativa, con ello pretendimos lograr una descripción holística, individual y dinámica de la experiencia de los estudiantes, cuyo propósito fue comprender e interpretar en la realidad vivida sus significados y las acciones llevadas a cabo, con la finalidad de crear nuevo conocimiento. Para ello se abordó mediante el estudio de caso'1, por investigar a profundidad a una sola o varias entidades como las familias, grupos, instituciones u otras entidades sociales. Entendiéndolas en su totalidad como seres integrales ${ }^{12,13,14 .}$ 
Por ser un estudio de caso se tuvo en cuenta los principios planteados por Menga Ludke e Marli Audef ${ }^{15}$, desarrollándose en tres fases ${ }^{16}$. Abierta o exploratoria, sistemática en términos de recolección de datos, análisis e interpretación y la elaboración del informe.

Los sujetos de investigación fueron 13 estudiantes mujeres, del III ciclo de enfermería de la asignatura Cuidados de Enfermería Materno Perinatal, grupo A, obtenidas por saturación, sus edades oscilaron entre 17 a 22 años; los criterios de inclusión fueron: que las estudiantes universitarios llevaran en forma regular la asignatura antes mencionada y hayan cumplido con la totalidad de las prácticas programadas. Se excluyeron los estudiantes irregulares.

El escenario lo conformó el aula del III ciclo "A" de la Escuela de Enfermería de la USAT, donde se entrevistaron a las estudiantes y se solicitaron sus diarios de prácticas para el análisis documental.

Como técnica de recolección de datos se utilizó la entrevista semiestructurada a profundidad ${ }^{14}$, previo consentimiento informado y el análisis documental de los diarios de práctica.

Posterior a la aprobación del proyecto, se inició la aplicación de las entrevistas y la revisión de los diarios de práctica. Para ello, las investigadoras citaron a las participantes en fecha y hora concretas que no les dificultaran la asistencia a clases teóricas o prácticas. Todas accedieron a colaborar y se les aseguró la privacidad y confidencialidad de sus discursos grabados mediante el uso de códigos.

Finalizada las entrevistas, se ejecutó el análisis de datos usando para ello el análisis de contenido 17, 18, 19, pasando por las etapas de preanálisis; codificación y por último la categorización.

Cabe mencionar que en la investigación se consideraron manifiesta que sólo una ética sustentada en una ontología los principios de la ética personalista, de Sgreccia, ${ }^{20}$ quien conveniente tomar como criterio de rigor científico: la puede pretender ser normativa. De igual manera se creyó credibilidad, la confirmabilidad, la audibilidad y transferencia ${ }^{21}$.

\section{Resultados, análisis y discusión}

Se obtuvieron los siguientes categorías y subcategorías: (1) Experiencia de una herramienta consolidada de práctica: (1.1) Para observaciones y anotaciones de la práctica. (1.2) Para el eguimiento en la consolidación de competencias. (1.3) Para estandarizar el lenguaje de enfermería. (1.4) Para autoevaluación del aprendizaje. (2) Registro de sentimientos personales: (2.1) Satisfacción frente a logros escritos. (3) Consolidación de la teoría con la práctica y viceversa.

Experiencia de una herramienta consolidada de práctica. Como las experiencias están determinadas por el conocimiento derivado de la observación, de la vivencia de un evento que sucede en la vida, referido al conocimiento procedimental (como hacer algo) según las expresiones de Reeve7, las anotaciones de datos en el diario de práctica los sujetos de estudio los consideraron útil. Este diario según ellos se constituye en una herramienta consolidada porque resulta ser una crónica objetiva y subjetiva que refleja las actitudes, los sentimientos y el aprendizaje cognitivo, en el transcurso de la experiencia práctica, en este sentido, la escritura de un diario supone ejercitar la reflexión ${ }^{22}$, reflexión que les conduce a escribir metas, procedimientos de su asignatura y también considerarla:

\section{Para observaciones y anotaciones de la práctica}

El estudiante de enfermería durante su práctica realiza observaciones al comentar como mira, habla, o actúa la otra persona a quien va a cuidar o el contexto que les rodea, estas observaciones pueden ser afirmativas o negativas. Cuando las 
observaciones son afirmativas, suelen ayudar a la persona a comunicarse sin necesidad de preguntas, enfoques o aclaraciones extensas, pues ayuda a empezar una comunicación. Cuando son negativas requiere hurgar en la situación o esperar el momento propicio para abordarlas. Las observaciones le ayudan al estudiante a definir con más claridad el significado de cualquier incidente clínico. Es así como el diario se convierte en una herramienta útil, luego en instrumento donde anotar sus observaciones y anotaciones de práctica, sistematizada y en una nutrida fuente de datos para que el estudiante repita las experiencias importantes y logre introspección en los pensamientos y acciones que constituyen la práctica ${ }^{22}$, tal como se evidencia en los discursos:

“... Ahí anoto todas las actividades de práctica, voy ejercitándome en las observaciones que nos guía la profesora en la sala de parto, en el alojamiento conjunto, cuando el bebé llora... los problemas que se presentan y todavía no lo hemos tocado en clase..." E9

“.... Anoto todo, a veces no me da tiempo, pero luego escribo todo lo que vi es muy importante y me sirve para seguir los problemas de salud de la puérpera como la veo, que observo en ella..." E7

Las estudiantes expresan como sus observaciones las pueden transcribir en su diario a pesar que todavía muchas de las temáticas que encuentran en la práctica no se han llevado a cabo en aula y lo más importante al no acabar de anotarlas revisan lo escrito y vuelven a escribir y de esta manera hay una secuencia lógica de su práctica.

Al respecto cabe mencionar que la epistemología de la práctica determina el mecanismos mediante el cual se genera y produce el conocimiento profesional: la reflexión-en-la-acción 22, 23. O sea, es la racionalidad práctica-reflexiva y no la técnica la verdaderamente útil en el enfrentamiento con las situaciones ambiguas e inestables de la práctica del cuidado y de la enseñanza del mismo.

Según Schön², el conocimiento profesional de la
Enfermera se ha caracterizado epistemológicamente como conocimiento práctico y ello, ha supuesto una reconsideración de la función de la enfermera como profesional del cuidado (del conocimiento requerido y su construcción, de sus habilidades), reconsideración que ha conducido, a su vez, a una transformación muy profunda en la conceptualización teórica de su formación inicial y permanente, en las estudiantes del tercer ciclo, este logro es progresivo por el nivel en que están pero al hacer uso de su diario de práctica para seguir la secuencia de lo observado en ella va camino de lograr una consolidación de la misma, aprécienos:

“.. mi diario de práctica es necesario e indispensable ya que nos corrobora en el día de prácticas para recordar y apuntar lo más importantes con la gestante y con el neonato, anoto lo que me llama la atención por ejemplo la palabra ictericia cuando he visto a un bebé muy amarillo..." E5

“...anoto las cosas más importantes y resaltantes que observo en mí práctica, para después recordarlas y estudiarlas, lo observado a la persona que mi profesora me asigno, para construir mi situación problema que lo registro, lo que la persona me dice, y sobre todo en el momento que entro en contacto por primera vez con la gestante... " $E 1$

“...hasta ahora me está ayudando mucho y es una guía...por ejemplo cuando tuve a mi cuidado a una madre adolescente... todos sus problemas, pensar que es menor que yo, y que problemas tiene; asi voy organizando mi situación problema..." E3

Los estudiantes expresan como hacen anotaciones importantes después que se les ha asignado a una determinada persona para su cuidado, o lo observado en toda una situación de enfermería como lo es el parto, sus anotaciones expresan lo que les llama la atención porque luego al configurar su situación problema traen a la memoria todo lo observado por primera vez y 
escrito por ellos mismos, así les resulta útil e indispensable.

\section{Para el seguimiento en la consolidación de competencias}

Consolidar competencias significa adquirirlas y poderlas aplicar cuando la situación de enfermería lo requiera, así una competencia involucra un conjunto de conocimientos, habilidades y actitudes necesarias para que un profesional desarrolle adecuadamente las funciones $y$ actividades que le son propias. Esta definición se estructura en tres grandes dimensiones como: conceptuales $o$ pensamiento crítico (conocimientos, toma de decisiones), interpersonales (actitudes, valores, etc.), técnicas (destrezas, habilidades) 24. Los sílabos de las asignaturas troncales en la Escuela de Enfermería, están configurados en competencias que los estudiantes deben alcanzar en el cuidado de la gestante y su neonato.

La competencia aprendida por parte de los estudiantes y escrita en un diario de práctica depende, en gran medida, de cómo el asesor de práctica equilibre la diversidad y la repetición de los contenidos del aprendizaje y de qué manera acompañe a sus estudiantes hacia delante y hacia atrás a manera de conocer el mundo en el campo práctico, 24 especialmente cuando la situación de salud está referida a adquirir competencias en el cuidado a la madre gestante y su bebé, como lo expresan las estudiantes:

“...nos ayuda para podernos dar cuenta de todo lo que hacemos de acuerdo a las exigencias $o$ competencias asignadas por la profesora en los días de práctica, de esa forma verificamos lo que nos falta por hacer cuando cuidamos a la persona o aun neonato...". E3

“...después de explicado en clase como debemos aplicar un procedimiento en el cuidado de la madre gestante o su bebé, en mi diario iba anotando el número de procedimientos que iba logrando en cada rotación para lograr las competencias de la asignatura...logre hacer cuatro maniobras de leopold, dos tactos vaginales, cinco baños al neonato, medir un promedio de siete alturas uterinas y presencie dos partos...igual vi lo que me falta, mis errores para mejorarlos... mis profesoras terminada la rotación nos revisan el diario y lo firman... El

“...se puede analizar lo escrito o realizado en el día, igual lo que hasta ese momento no había hecho con la madre asignada para poner metas, hacerlas y ayudarla... de mi parte esta brindar un mejor cuidado..." ElO

“... me permite el reforzamiento de los conocimientos cuando reviso el diario en mi casa para luego ver en qué cosas tengo que estar más segura y poder ayudar más a la gestante..." E8

El diario de práctica facilita anotar el número de actividades terapéuticas, sin embargo también sirve para anotar los errores encontrados en la realización de actividades, y también verificando en el diario que hay competencias que no se han cumplido como por ejemplo la observación de una cesárea, es de suponer las dificultades con respecto a la habilidad en el manejo de estos procedimientos, más no hacen mención del aspecto actitudinal en forma concreta, así demuestran que la consolidación de sus competencias se logra verificando el cumplimiento de los objetivos sólo basado en el número de procedimientos, igualmente hacen referencia como les ayuda cuando el diario es revisado por su profesora o por ellos mismos para reforzar la seguridad en sí mismos para su próxima experiencia práctica, es así como se evidencia en el diario una sugerencia de la profesora para.

\section{Para estandarizar el lenguaje de enfermería}

El lenguaje estandarizado25,26, favorece el desarrollo del conocimiento enfermero, proporcionando información para la formulación en la toma de decisiones. Cuando se usa un 
lenguaje enfermero común facilita el acercamiento hacia la excelencia profesional que contribuye a elevar el nivel del estudiante de enfermería en todos los campos y funciones de enfermería, asistencia, gestión, docencia e investigación, que disminuya la variabilidad de los cuidados, y mejore la comunicación en los distintos ámbitos, asumiendo así la responsabilidad pertinente de los mismos y documentar la contribución de la enfermera a la salud de las personas tal como se evidencia en los discursos:

“...Me he dado cuenta que dentro de la práctica los profesionales hablan el mismo lenguaje, aquello que no logro comprender, los apunto en mi agenda y luego los reviso, ya que como futura profesional de salud necesito estar consciente de la importancia del lenguaje utilizado en los hospitales o la comunidad de esta manera me ayuda a evaluar y reforzar mis cuidados"..." E2

Dentro de estos párrafos proporcionados por los estudiantes da certeza que el diario de práctica sirve también como una herramienta para estandarizar el lenguaje de enfermería y la terminología propia de los profesionales de enfermería y utilizados en los servicios de hospitalización, aún desconocida incluso no la comprenden y esto es cierto pues están todavía en el tercer ciclo de estudios de su carrera profesional, sin embargo tienen el deseo de aprenderlas y por eso las anotan para utilizarlo en el momento oportuno, ayudando al estudiante a crecer profesionalmente, teniendo la capacidad de expresarse en forma profesional, y si no recuerdan algún termino, tienen la facilidad de ir al diario retroceder y encontrar la terminología en el momento mismo de la necesidad, solucionando así el problema presente.

“.. para la asignatura de materno perinatal me ayuda a tener en cuenta los términos, que se utilizan en la práctica, ya que muchos de ellos son nuevos para mí, y son importantes para entender a los médicos, obstetrices y enfermeras al momento de la evaluación de la madre o del niño y nosotros podamos entender lo que explican y los procedimientos que hacen..." E5

“... apunto los datos importantes como por ejemplo terminología que desconozco, aumentando así mi aprendizaje, estas nuevas palabras pueden ser de mucho interés para la ejecución de enfermería en la práctica... también trato de familiarizarme con todo el lenguaje empleado en el hospital entre los profesionales con los que practico...." E4

De acuerdo a lo expresado por los estudiantes valoran lo importante que es asumir y practicar un lenguaje enfermero, términos que los llegan a escribir en su diario de práctica, algunos los desconocen o resultan ser nuevos los asumen para mejor entendimiento del común léxico del equipo de salud que atiende a la madre y el niño y sobre todo porque aciertan a expresar que pueden entenderles y ya no se sentirán distintos de los demás miembros del servicio hospitalario.

También expresan como el apuntar les ayuda a ir acrecentando este lenguaje porque aprenden a utilizarlo y no sólo está el hecho de apuntarlo, y es que el lenguaje común permitirá caminar hacia la excelencia de los cuidados profesionales para que se pueda asumir la total responsabilidad de los mismos y documentar la contribución de enfermería en la salud de las personas. Del mismo modo permitirá definir la profesión, normalizar la nomenclatura de fenómenos, intervenciones y resultados que permita desarrollar sistemas de información o desarrollar conocimientos científicos ${ }^{27}$. Al respecto los estudiantes también valoran el diario de práctica.

\section{Para autoevaluación del aprendizaje}

La autoevaluación del aprendizaje permite que el estudiante del tercer ciclo de enfermería sea más responsable, pues aprende a asumir la responsabilidad de sus actos cuando debe decidir los criterios que le resultan más importantes, los objetivos que quiere alcanzar y juzgar en qué 
medida los ha logrado28 y la autoevaluación resulta más objetiva cuando las cosas están escritas y se comprueba resultados así lo expresan los estudiantes:

"...El diario me sirve de mucho para autoevaluarme, lograr objetivos durante la práctica, y luego darme cuenta también de los logros a los que llegue durante ese día, la felicitación de mi profesora después de un procedimiento me sirve de mucho para seguir evaluándome como estudiante de enfermería ..." E6

La autoevaluación consiste en la valoración, por parte del propio estudiante del rendimiento educativo que ha obtenido, realizado de manera individual o colectivo. ${ }^{29}$

Es decir la autoevaluación se convierte en la base de la regulación permanente del autoaprendizaje y la enseñanza, constituyéndose en un objetivo educativo y por ello un principio rector de alguno de los procesos de la enseñanza universitaria en enfermería.

Igualmente, para los estudiantes autoevaluarse es ver si los logros apuntados en su diario han sido ejecutados y al comprobarlo se experimentan alegría al recibir la felicitación correspondiente por parte de su profesora.

“.. nos permite plasmar todos los procesos realizadas durante la práctica, así evaluó mis procedimientos y mi actuar dentro o fuera del hospital... Una vez por ejemplo tuve práctica fuera del hospital en un pueblo joven y encontré en una casa un niño de 6 días de nacido y lo vi muy amarillo, al observarlo, me di cuenta que se trataba de una ictericia postnatal, aconseje a su madre para lo que lo llevase al centro de salud, luego cuando le hice el felicitaron y lo puse como un comentario en mi comentario a mi profesora $y$ compañeras me autoevaluación diaria..." E9

Los estudiantes al autoevaluarse están realizando la reflexión desde la acción experimentadas en la práctica ${ }^{29}$ es decir, llevan a cabo sin darse cuenta un proceso de análisis acerca de la misma partiendo de lo escrito en su diario y de los resultados obtenidos, de una manera más ponderada, ya que no se hayan solicitados por las demandas de la inmediatez de las situaciones prácticas, de esta forma el estudiante va reconstruyendo y comprendiendo retrospectivamente sus procesos de reflexión en la práctica.

La autoevaluación, ${ }^{29}$ que realizan se centra únicamente en las características de la situación o contexto del problema, se cuestionan también los procedimientos llevados a cabo para formular el problema y determinar su naturaleza, la formulación de objetivos, la selección de los cursos de acción realizada, más no se expresan el registro de equivocaciones u omisiones...etc., tan propio de un manera a comprenderse mejor $y$ comprender la estudiante de tercer ciclo que les conduciría de alguna realidad donde llevaron a cabo la acción para rectificar y crecer; entonces aquello en que puede potenciar más o tener habilidad queda ciertamente detenido y el mejoramiento personal es más lento, sin embargo resulta valioso la autoevaluación de los estudiantes porque supone una meta reflexión en torno al conocimiento de la acción y la reflexión en la acción.

“.. plasmo lo más importante que realizo en la práctica: metas, tareas asignadas, investigaciones, las cuales me van a permitir evaluarme así refuerzo mis conocimientos para las próximas veces que debo realizar alguna tarea en el hospital y actuar con responsabilidad, sentirme segura de hacerlo sin equivocarme..." E4

Los discursos de los estudiantes expresan el valor que le dan a la autoevaluación en la adquisición de sus competencias, sintiéndose satisfechos por los logros obtenidos, También les permite determinar y revalorar los esfuerzos realizados día tras día sintiendo que su actuar diario es productivo, dándoles mayor seguridad en los procedimientos terapéuticos que deben realizar a la madre o al 
neonato sin temor a la equivocación e ir disminuyendo la falta de conocimientos propios de la asignatura y es que la autoevaluación a menudo, es una tarea muy difícil. Pues el estudiante debe formular sus criterios de evaluación, decidir según las normas que tiene para sí mismo, experimentar como va su avance, vislumbrar las dificultades y obstáculos encontrados, pues el mismo es el autor de su aprendizaje28,29. Por otro lado un diario de práctica también es utilizado por los estudiantes de enfermería como un:

\section{Registro de sentimientos personales}

Los sentimientos ${ }^{7}$ son polarizaciones que hace nuestra mente de los hechos, su origen es el resultado del movimiento de las cargas emocionales a las cuales nuestra mente se ve sometida por la variación del medio. La mente establece el objetivo y los hechos fomentan o contrarrestan su consecución y preservación. La variación del estado preferente que hace la mente del objetivo, induce en ella el sentimiento que la motiva a actuar.

Según esta definición los sentimientos para los estudiantes escritos en el diario de práctica permiten que las emociones resultantes de sus experiencias en la práctica de la asignatura materno perinatal, puedan encontrarse como sentimientos subjetivos que derivan del propio pensamiento y las propias percepciones. Aquí cada uno de ellos logran entender que los sentimientos no tienen un significado específico es decir: no son correctos, erróneos, buenos o malos, aunque pueden ser agradables o desagradables, todo ello depende de la forma en que estos son registrados durante las horas de práctica. Es así como emerge la subcategoría:

\section{Satisfacción frente a logros escritos}

La actitud hacia la profesión se desarrolla a partir de motivaciones, entre otras que inclinan positiva o negativamente al individuo30, ocurre en ocasiones que una vez dentro de ellas se producen frustraciones o también afirmaciones lo que conlleva al estudiante a la deserción o a la continuidad de los estudios.

Estas afirmaciones del autor coinciden con los discursos de los estudiantes quienes al leer los logros alcanzados en su diario de práctica reafirman su satisfacción experimentando que no se equivocaron al elegir ser enfermeras:

“...Los cuidados y procedimientos que son realizados a las personas a mi cuidado me permiten saber que lo que elegí está bien, es decir ser enfermera, cuando leo lo ganado en estos procedimientos me siento muy bien..." E2

“... Después de cada práctica, de ver todo lo hecho, me doy cuenta que me gusta todo lo que realice, lo me preguntan siempre si estoy contenta en la que estudio y que fue una buena decisión, mis padres universidad, ahora respondo con más firmeza que sí y sin dudas..." E3

Las expresiones de los estudiantes están llenas de satisfacción con la madre gestante a su cuidado, vivencian el gusto por la profesión de enfermería y afirman categóricamente que eligieron bien, desechando la idea o la tipificación de la enfermera como aquella persona que sólo aplica inyectables, por otra parte se siente realizadas al cuidar a la persona humana y todo con el simple hecho de leer lo escrito en su diario de práctica.

Sin duda el hecho de escribir y luego al leer, se comprueba la consonancia con la realidad. Lo cual produce en la interioridad del estudiante de enfermería una gran satisfacción que les lleva a expresar que la decisión que tomaron de elegir la carrera de enfermería no fue equivocada, esto también visiona que en ellos existía la posibilidad de dudar si eligieron bien o no, sin embargo al ojear su diario y en la comprobación de un hecho, esa decisión voluntaria y libre realizada no fue equivocada.

\section{Consolidación de la teoría con la práctica y viceversa}


Consolidar, 22 significa dar firmeza y solidez a algo; en la intención de que la teoría y la práctica logre su fortalecimiento como metodología en la educación y que garantice las relaciones positivas entre los estudiantes y los que deseen estudiar, para permitirles enriquecer conocimientos donde lo estudiado en clase tenga coherencia con lo que se encuentran en la práctica común y que no se den teorías inalcanzables en la práctica, así esta consolidación se apoya en la ética educacional que debe ser sincera, la cual contempla a su vez una educación en valores.

Por otro lado, la consolidación se dará mediante las aportaciones de la práctica en enfermería en las que se describen los elementos del conocimiento práctico asumiendo reflexión acción ${ }^{30}$.

Esta reflexión acción hace generar en los estudiantes puntualizaciones en metas personales que además de escribirlas tienen la connotación de ser reflexivas donde su racionalidad les hace vislumbrar a quien se enfrentan en el desarrollo de la práctica, de esta manera actúan en forma independiente con ánimo investigador y creador cuando se disponen brindar el cuidado a las madres y neonatos, como ellos mismos lo dicen:

“...Dentro de las clases teóricas entre los temas hablamos sobre mejorar el trato con las personas y lo escribi en mi diario, sobre todo cuando atendemos a madres embarazadas adolescentes que necesitan de nosotros para estar tranquilas ya que muchas de ellas son madres solteras, y los problemas a los que se enfrentan son físicos $y$ psicológicos, intento atenderlas mejor...también saber que debo estudiar más, para ir preparada a la práctica... "El

Las clases teóricas para ser aplicados a la práctica son escritas por los estudiantes en su diario de práctica, por ello, el conocimiento profesional de la enfermera está caracterizado como conocimiento práctico y además, ha supuesto una reconsideración de la función de la enfermera como profesional del cuidado, es decir del conocimiento requerido y su construcción, de sus habilidades, reconsideración que ha conducido, a su vez, a una transformación muy profunda en la conceptualización teórica de su formación inicial y permanente ${ }^{30}$.

En los estudiantes el camino de la transformación se está dando pues reconocen que las gestantes adolescentes con problemas y que los necesitan teniendo por escrito como deben tratarlas y hacer el contraste con la realidad optan por mejorar y estudiar más. Por otro lado, la práctica está cimentada en modelos y teorías enseñadas por sus profesoras en aula que forma parte de la meta de su diario de práctica tal como se evidencia y el discurso:

“...Con ayuda de la profesora voy mostrando seguridad y confianza a la hora de realizar cualquier procedimiento, dentro de una clase hablamos sobre los pensamientos de las teóricas Martha Rogers, Orem, Peplau y muchas más, trabajo con Leninger y voy anotando los aspectos culturales que observo en la madre y el niño, esta teórica me permitió interactuar con la madre que requería de mis cuidados y sobre todo brindarles mi respeto y cariño..." E2

Los estudiantes aluden que la seguridad y confianza vine de la experiencia de sus profesoras, en este sentido. la seguridad y confianza son valores humanos, que se caracterizan porque a través de ellos se logra la madurez en las relaciones humanas, implica también estabilidad, respeto, amor ya que todos requieren que alguien confíe en uno mismo contribuyendo así a que la persona crezca en el ámbito personal, profesional y espiritual, 30 como lo expresan las estudiantes de enfermería al referirse a sus profesoras, seguridad y confianza que luego de ser anotados los van aplicando en la práctica producto de ideas o teorías que han recibido en las clases y al estar delineadas en el diario les permiten contrastar con los aspectos 
culturales que encuentran en las personas a su cuidado.

Por otro lado el proceso reflexivo llevado a cabo en la asignatura materno perinatal les motiva a expresar:

“...Este ciclo de materno perinatal, creo que estoy tomando mayor conciencia de las cualidades que debe tener una enfermera al momento de la atención y son importantes rescatar los valores que guían el comportamiento de cada una de nosotras... en alguna ocasión se dio en clase el tema de Cualidades de la enfermera, de ahí aprendí mucho...y escribí que tenía que estudiar, tener más iniciativa..." E13

"Una vez tuve que observar a una madre dando de lactar a su niño y con lo aprendido en el aula me di cuenta que la técnica empleaba por la madre era incorrecta, así constate como lo aprendido en aula se convierte en importante al momento de la práctica...me sentí contenta porque enseñe a la madre la técnica adecuada para que le dé a su niño la leche materna...y anote este logro....pequeño pero muy importante para mi..." E8

Los estudiantes al tomarse un tiempo para reflexionar intentan describir la importancia de esta consolidación práctica tiene para ellas, así identifican momentos o situaciones estudiadas en sus clases teóricas, como son las cualidades que debe tener una enfermera, que les condujo quizá a examinarse asumir a conciencia como se estaba realizando su trabajo, además contrastar la teoría con la práctica, de esta forma se dan cuenta el desequilibrio que hay en la realidad, y lograr corregirla como es el hecho de la forma como una madre da de lactar a su bebé creando en ellas esa conciencia de investigación, así como se evidencia en el diario, por lo que saben que encontraran situaciones que no les serán desconocidas si lo han revisado anteriormente en clase.

Estas acciones encontradas en práctica son plasmadas en su diario de diferentes maneras, a veces solamente colocando las actividades que se realizaron, o leer para recordar esas situaciones presentadas, por tal motivo un diario puede ser utilizado tanto en la formación teórica como en las prácticas hospitalarias 0 comunitarias. Las estudiantes de enfermería de esta investigación valoran así lo obtenido en práctica cada vez que realizan o consolidan sus actuaciones en forma positiva o asertiva, manifestadas en los discursos anteriores, encontrando en ellas ese fortalecimiento científico que buscan y que es una exigencia de la universidad y deben ser imitados por todas las asignaturas troncales.

\section{Conclusiones}

1. La experiencia en el uso del diario de práctica para las estudiantes del tercer ciclo de enfermería de la asignatura Cuidados de Enfermería Materno Perinatal, grupo A, resultó ser una experiencia rica, evidente y útil exigido por sus profesores para llegar a consolidar sus competencias, que si bien es cierto, demostraron aspectos de conocimientos y habilidades no se evidenció en la parte escrita , aunque si en los discursos, los aspectos actitudinales, dígase de paso aspecto difícil de escribir y que requiere ser considerados en los diarios para el momento de autoevaluarse y se afirme a futuro que el logro de la competencia es completo.

2. Durante sus experiencias prácticas las estudiantes del tercer ciclo de enfermería de la Universidad Católica Santo Toribio de Mogrovejo hacen uso del diario de campo, cada vez que van a la práctica, de tal manera que lo consideran como una herramienta consolidada, donde hacen anotaciones de sus observaciones; escriben como van consolidando sus competencias, se interesan por escribir términos que desconocen y les llama la atención al ser escuchados y todavía no dominan, además su diario les permite autoevaluarse, reconociendo sus errores y asumiendo con responsabilidad lo escrito por sus profesoras como observaciones a cumplir. 
3. La experiencia de usar un diario de práctica también ayuda a los estudiantes del tercer ciclo de enfermería a registrar sus sentimientos personales, especialmente la satisfacción por los logros obtenidos durante sus prácticas preprofesionales.

4. Los sujetos de estudio también experimentaron cómo su diario de práctica, les favorece en la consolidación de la teoría con la práctica y viceversa, las cualidades que deben tener y cuándo existe un desequilibrio entre la teoría y la realidad observada y cómo pueden intervenir.

\section{Bibliografía}

1. Serrano P. Evaluación de las Prácticas Clínicas de la Asignatura De Enfermería Comunitaria a través del Portafolio. Oficina de Convergencia Europea. Madrid; 2007. [Citado el: 13-04-2008]. Disponible en:

http:/ / www.adi.uam.es/europea/archivos/Convoc atorias\%20UAM/memoria\%20proyecto\%20Portafol ios\%202007.pdf.

2. FarguesG, et al. Diario Reflexivo De Prácticas Clínicas: Percepción De La Experiencia De Estudiantes De Enfermería.2006. [Citado el: 1304-2008]. Disponible en: http://www.enfermeria 21 .com/pfw_files/cma/revistas/metas/2007/98/ enportada98.pd

3. MorenoM, et al. El diario Reflexivo: Herramienta Pedagógica innovadora en las Prácticas de Enfermería. Barcelona; 2007. [Citado el: 13-042008]. Disponible en: http://www.uem.es/myfiles/pageposts/Memoria_ 1_premio.pdf

4. Ortiz E. Las Técnicas de Avalúo del Diario Reflexivo o del Portafolio y el aprovechamiento académico de estudiantes de Educación. $\mathrm{N}^{\circ} 18$. Puerto Rico: Recinto de Rio Piedras; 2002.

5. Sánchez A. Enfermería comunitaria. España: Mc GRAW-HILL; 2000
6. García L. El sistema de las virtudes Humanas. Barcelona: Mc GRAW-HILL; 1999.

7. Reeve, J. Motivación y Emoción. Mexico: McGRAW-HILL; 2002.

8. Marrineer A, Alligood M. Modelos y Teorías de Enfermería. 4ta edición: Madrid. Harccort; 2000.

9. Papalia ED, et al. Desarrollo humano. 4ta Edición. Colombia: Interamericana; 1994.

10. López,L, et al. Revista Electrónica de Enfermería: Una Experiencia sobre la Utilización del Diario Reflexivo como instrumento de seguimiento y evaluación de las Prácticas Hospitalarias del Alumnado de Enfermería., [Revista en internet] 2008 [acceso el 20 de agosto del 2008]. [Actualiza en febrero del 2008;]. Disponible en:

http://www.um.es/ojs/index.php/eglobal/article/ view/1351/2911.

11. Polit $D$, et al. Investigación científica en ciencias de la salud: principios y métodos. 6ta Edición. México D.F: McGraw-Hill Interamericana; 2000.

12. Rodríguez G. Metodología de la investigación cualitativa. Granada: ALJIBE S.L; 1996.

13. Canales F. Metodología de la investigación: Manual para el desarrollo de personal de salud. 2da edición. Washington: D.C: OPS; 1994.

14. Nisbet J, Watt J. Conducting Small-scale investigations in educational management. London. Paul Chapman Publishing ang Ltd; 1978.

15. Ludke M, Marli A. Pesquisa em Educação: Abordagens Qualitativas. 3era edición. São Paulo. 1988.

16. Del Valle F. Análisis Documental. Madrid: Universidad Complutense de Madrid; 2001. 
17. Lincoln YS, Guba EG. El inquiri naturalista. Beverly Hills. Sage Publications; 1985.

18. Iguiñez V. Etapas de los análisis de los datos. Investigación cualitativa. Fundamentos, técnicas y métodos. Chile; 2004.

19. Stake RE. The case study method in scial inquirí. Educational Researcher. Vol 7. Ilinois: Springer Netherlands; 1978.

20. Sgreccia E. I Congreso Internacional de Bioética. Perú: Universidad Católica Santo Toribio de Mogrovejo; 2008. Disponible en: http://www.usat.edu.pe/investigacion/cib/congre sobioetica/descargar.htm.

21. Pérez G. Investigación cualitativa: retos e interrogantes II. Técnicas y análisis de datos. 3era Edición. Madrid : La Muralla; 2000.

22. Schon D. La formación de profesionales reflexivos. Hacia un nuevo diseño de la formación y el aprendizaje en las profesiones. Madrid: Piados MEC; 1992.
23. Maiceira B. Nivel de vocación por la especialidad de enfermería en los estudiantes del 2do año. Cuba: Revista Cubana de Enfermería; 1999.

24. García J, Rodríguez G. Metodología de la Investigación Cualitativa. 1996.

25. Potter P. Fundamentos De Enfermería. 6ta Edición. Barcelona: ELSEVIRER; 2015.

26. Barberá E. El Constructivismo en la Práctica. 4ta Edición. Barcelona: Editorial Laboratorio Educativo; 2007.

27. Mccloskey. Intervenciones De Enfermeria (Nic). 4ta Edición, Madrid: Elsevier; 1997.

28. Notoria A. Aprendizaje Centrado en el Alumno. Metodología Para Una Escuela Abierta. España: Nancea S.A. De Ediciones; 2009.

29. Castillo S. Compromisos de la Evaluación Educativa. Madrid: Person Educación S.A; 2002.

30. Haydon G. Enseñar en valores. Un nuevo enfoque. Madrid: Morata; 2003. 